\title{
Three-Dimensional Numerical Simulation of Bubble Dynamics in Microgravity under the Influence of Nonuniform Electric Fields
}

\author{
Tai Wang ${ }^{1} \cdot$ Hui-Xiong $\mathrm{Li}^{1} \cdot$ Jian-Fu Zhao ${ }^{2}$
}

Received: 26 May 2015 / Accepted: 26 January 2016 / Published online: 7 March 2016

(C) Springer Science+Business Media Dordrecht 2016

\begin{abstract}
A three-dimensional VOSET method is used along with the adaptive mesh refinement (AMR) method to simulate the behaviors of a bubble departing from the outside wall of a horizontal square-cross-section tube in microgravity under the influence of nonuniform electric fields. The effects of gravity, electric field intensity, fluid permittivity, and bubble initial position on the bubble detachment and rising are investigated and analyzed. Computational results show that the gravity and electric fields have significant influences on the bubble detachment and rising velocity and rising trajectory. Decrease in gravity results in the decrease in the buoyancy exerted on the bubble, considerably mitigating the rising capability of the bubble and delaying the bubble detachment. Imposing a nonuniform electric field, which exhibits physically the strongest intensity in regions near the tube wall, can supply an additional driving force as a replacement of the buoyancy to accelerate the bubble detachment and rising. It is also shown that a larger electric field intensity or larger ratio of liquid permittivity to gas permittivity leads to a larger deformation, easier detachment, and larger rising velocity, of the bubble. The nonuniformity of the electric fields can also affect the bubble motion trajectory and result in the asymmetric deformation of the bubble.
\end{abstract}

Hui-Xiong Li

huixiong@mail.xjtu.edu.cn

1 State Key Laboratory of Multiphase Flow in Power Engineering, Xi' an Jiaotong University, Xi'an, 710049, China

2 Key Laboratory of Microgravity (National Microgravity Laboratory) / CAS; Institute of Mechanics, Chinese Academy of Sciences (CAS), Beijing 100190, China
Keywords Adaptive VOSET method · Nonuniform electric fields $\cdot$ Bubble detachment $\cdot$ Microgravity . Numerical simulation

\section{Introduction}

Electrohydrodynamics (EHD) (Castellanos 1998) is a cross discipline of fluid mechanics and electrodynamics, which mainly addresses fluid flows under the influence of electric fields. Multiphase electrohydrodynamic flows, as a branch of EHD flows, is frequently encountered in many applications such as inkjet printing (Minemawari et al. 2011), electrowetting (Lee et al. 2002), electrospraying (Jaworek and Sobczyk 2008), and enhancing boiling heat transfer (Di Marco 2012a), and so on. Applying an electric field on bubbles, in which the electric force acts on the phase interface, is a good method to control the bubble deformation and bubble motion. Thus these studies have attracted more and more attentions from researchers.

Over the last decade, amount of experimental and numerical work have been done to investigate bubble dynamics in terrestrial gravity under the influence of electric fields. Dong et al. (2006) reported experimental investigations of bubble behaviors in a uniform electric field, and analyzed the effects of different electric field intensities on the bubble growth, deformation, detachment and break-up. Bari and Robinson (2013) performed similar experimental research and calculated the variation of the buoyancy, capillary force and other forces acted on the bubble. Jalaal et al. (2010) constructed a nonuniform electric field using the horizontal and vertical electrodes, and investigated the influence of the nonuniform electric fields on bubble behaviors, and the experimental results showed that the nonuniform electric field can affect the motion trajectory of the bubble. 
Gao et al. (2013) experimentally studied the growth of a single R113 vapor bubble under nonuniform electric fields which were built by a vertical needle and a horizontal heating plate, and observed that the nonuniform electric fields would restrain the bubble growth. Mahlmann and Papageorgiou (2009) numerically simulated a bubble rising under a uniform electric by a two-dimensional level set method. Wang et al. (2015) used VOSET method to study the influence of electric field intensity, fluid permittivity, viscosity, and surface tension force on bubble deformation and rising under uniform electric fields. Sunder and Tomar (2013) used a CLSVOF method to simulate bubble formation from submerged needles under nonuniform electric fields, and the numerical results showed that imposing nonuniform electric fields is a good method to control the bubble departure volume and departure frequency.

In microgravity environments, the decrease in gravity leads to the decrease in buoyancy, which brings increasing difficulty in the detachment of bubbles attached to a wall. The most important example is bubbles growing on a heated wall in microgravity (Straub 2001; Di Marco 2003a; Kim 2003, 2009; Ohta 2003; Zhao 2010). Xue et al. (2011), among many others, experimentally studied nucleate pool boiling of FC-72 in normal gravity and microgravity conditions, and the results showed that the heat fluxes in microgravity are low due to the fact that large bubbles remain attaching to the heating wall for a long time. In order to enhance nucleate boiling heat transfer in microgravity, developing an effective method accelerating bubble detachment is necessary. Then some researchers have tried adding an electric force on the bubble to affect the bubble motion. For example, Herman et al. $(2002,2004)$ and Iacona et al. (2006) presented the experimental investigations of bubble growth and detachment in microgravity under the influence of electric fields. Di Marco et al. (2003b, 2007, 2012a) performed similar experiments using another electric configuration, and the experimental results showed that choosing an appropriate electric field can promote the bubble detachment. Thus, it is very significative to further investigate and understand the influence of electric fields on bubble dynamics under various microgravity conditions. Up to now, there are only much small amounts of experimental investigations of EHD flows conducted in microgravity, as well as the numerical work, especially for the three-dimensional numerical simulations.

In this paper, a three-dimensional VOSET method (Sun and Tao 2010; Wang et al. 2015) is used along with the adaptive mesh refinement (AMR) techniques (Theodorakakos and Bergeles 2004; Yu and Fan 2009) to study the influence of nonuniform electric fields on bubble departing from the outside wall of a horizontal square-cross-section tube in microgravity. The AMR techniques based on octree method are specially employed to refine the grids around the twophase interface, in order to, on the one hand, enhance the calculation accuracy and, on the other hand, reduce the computational time. The electric force model for the perfect dielectric fluids is used to calculate the electric force acted on the two-phase interface. Using the numerical framework, we investigated the influence of gravity, electric field intensity, fluid permittivity, and bubble initial position on the bubble dynamics under nonuniform electric fields. As a part of the ground-based advance research of the project of SOBER-SJ10, which is one of the selected experiments of the China space mission SJ-10 ( Hu et al. 2014), more attentions will be paid on the effect of the electric fields on bubble dynamics in the microgravity conditions.

\section{Physical Model}

The 3D physical model in the present study is shown in Fig. 1a. The electric field is applied between two horizontal electrodes, in which a small square tube (high electrode) locates in the center of a bigger square tube (ground electrode). Initially, a spherical bubble with radius $R=0.004 \mathrm{~m}$ attaches to the outside wall of the small horizontal square tube. Since the bubble departs from the top wall of the horizontal tube, $1 / 2$ domain $(\mathrm{z}>0)$ of the physical model is used as the computational domain shown in Fig. 1b. The domain size and tube size are considered to be $L_{x}=2 L_{y}=$ $2 L_{z}=0.1 \mathrm{~m}$ and $l=0.02 \mathrm{~m}$, respectively.

Fig. 1 Schematic illustrations of physical model and computational domain

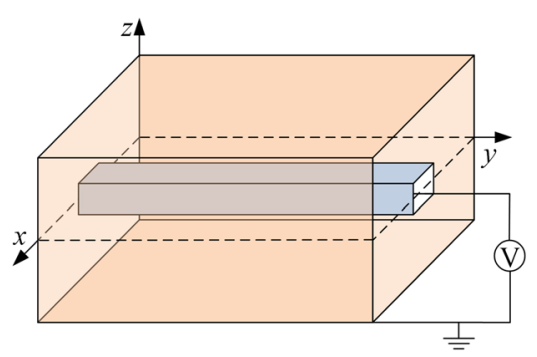

(a) physical model

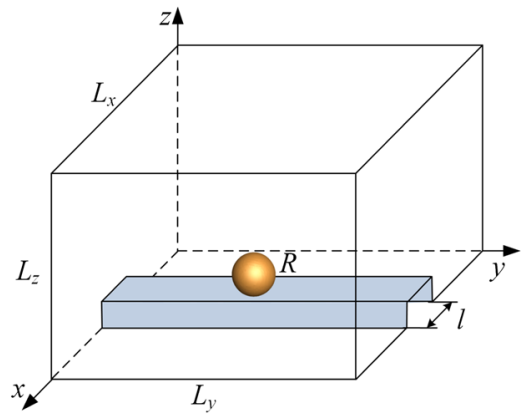

(b) computational domain 


\section{Numerical Formulations}

\section{VOSET Method}

In this paper, a coupled volume-of-fluid and level set (VOSET) method, which can keep mass conservation and accurately calculate surface normal and curvature, is used to capture the two-phase interface. In VOSET method, PLIC algorithm (Youngs 1982; Annaland et al. 2005) is implemented to solve the advection equation of the volumeof-fluid function $C$ defined as the volume fraction of gas in a cell.

$\frac{\partial C}{\partial t}+\boldsymbol{u} \cdot \nabla C=0$

After obtaining the value of $C$, a geometric method (Wang et al. 2009) is used to calculate the level set function $\phi$ which is adopted to compute the fluid properties.

$\rho=\rho_{l} H(\phi)+\rho_{g}(1-H(\phi))$

$\mu=\mu_{l} H(\phi)+\mu_{g}(1-H(\phi))$

$\frac{1}{\varepsilon}=\frac{H(\phi)}{\varepsilon_{l}}+\frac{(1-H(\phi))}{\varepsilon_{g}}$

where $\rho, \mu, \varepsilon$ are the density, viscosity, and relative permittivity, respectively. The subscripts $l$ and $g$ refer to the liquid and gas, respectively. $H(\phi)$ is the Heaviside function which is written as:

$H(\phi)= \begin{cases}0 & \text { for } \phi<-\gamma \\ \frac{1}{2}\left[1+\frac{\phi}{\gamma}+\frac{1}{\pi} \sin \left(\frac{\pi \phi}{\gamma}\right)\right] & \text { for }|\phi| \leq \gamma \\ 1 & \text { for } \phi>\gamma\end{cases}$

where $\gamma$ is the width of the smoothed region and equals $1.5 \Delta, \Delta$ is the least grid size.

\section{Fluid Flow Equations}

The governing equations described incompressible fluid flows are the mass and momentum conservation equations which can be expressed as:

$\nabla \cdot \boldsymbol{u}=0$

$$
\begin{aligned}
\rho\left(\frac{\partial \boldsymbol{u}}{\partial t}+\boldsymbol{u} \cdot \nabla \boldsymbol{u}\right)= & -\nabla p+\rho \boldsymbol{g}+\nabla \cdot\left[\mu\left((\nabla \boldsymbol{u})=(\nabla \boldsymbol{u})^{\mathrm{T}}\right)\right] \\
& +\boldsymbol{F}_{\sigma}+\boldsymbol{F}_{e}
\end{aligned}
$$

where $\boldsymbol{u}$ is the velocity vector, $p$ is the pressure, and $g$ is the gravitational acceleration in which the terrestrial gravity $g_{0}=9.81 \mathrm{~m} / \mathrm{s}^{2} . \boldsymbol{F}_{\sigma}, \boldsymbol{F}_{e}$ are the surface tension force and electric force, respectively.
A continuum surface force (CSF) model (Brackbill et al. 1992) is used to calculate the surface tension force.

$\boldsymbol{F}_{\sigma}=-\sigma \kappa \delta(\phi) \nabla \phi$

where $\sigma$ is the interface tension coefficient, $\kappa=\nabla$. $(\nabla \phi /|\nabla \phi|)$ is surface curvature, and $\delta(\phi)$ is the Dirac distribution function which is written as:

$\delta(\phi)= \begin{cases}0 & \text { for }|\phi|>\gamma \\ \frac{1}{2 \gamma}\left[1+\cos \left(\frac{\pi \phi}{\gamma}\right)\right] & \text { for }|\phi| \leq \gamma\end{cases}$

\section{Electric Field Equations}

In order to calculate the electric force on the bubble, it is necessary to solve the electric field distribution. For a perfect dielectric material, the electric field equation (Paknemat et al. 2012) can be written as:

$\boldsymbol{E}=-\nabla \psi$

$\nabla \cdot\left(\varepsilon_{0} \varepsilon \nabla \psi\right)=0$

where $\boldsymbol{E}$ is the electric field, $\psi$ is the electric potential, and $\varepsilon_{0}$ is the vacuum permittivity.

After calculating the electric field, the electric force $\boldsymbol{F}_{e}$ can be solved by the following formula.

$\boldsymbol{F}_{e}=-\frac{\varepsilon_{g} \varepsilon_{0}}{2} E^{2}\left(\frac{\varepsilon_{l}}{\varepsilon_{g}}-1\right) \delta(\phi) \nabla \phi$

\section{Boundary Conditions}

For the computational model (Fig. 1b), the following boundary conditions for solving the fluid flow equations and electric field equations are used.

$$
\begin{aligned}
& x=0: u=v=w=0, \psi=0 ; \\
& x=L_{x}: u=v=w=0, \psi=0 ; \\
& y=0: u=v=w=0, \frac{\partial \psi}{\partial y}=0 ; \\
& y=L_{y}: u=v=w=0, \frac{\partial \psi}{\partial y}=0 ; \\
& z=0 \text { and } x<0.5\left(L_{x}-l\right): \frac{\partial u}{\partial z}=\frac{\partial v}{\partial z}=0, \\
& w=0, \frac{\partial \psi}{\partial z}=0 ; \\
& z=0 \text { and } x>0.5\left(L_{x}+l\right): \frac{\partial u}{\partial z}=\frac{\partial v}{\partial z}=0, \\
& w=0, \frac{\partial \psi}{\partial z}=0 ; \\
& 0.5\left(L_{x}-l\right) \leq x \leq 0.5\left(L_{x}+l\right) \text { and } \\
& z \leq 0.5 l: u=v=w=0, \psi=\psi_{0} ; \\
& z=L_{z}: u=v=w=0, \psi=0 ;
\end{aligned}
$$




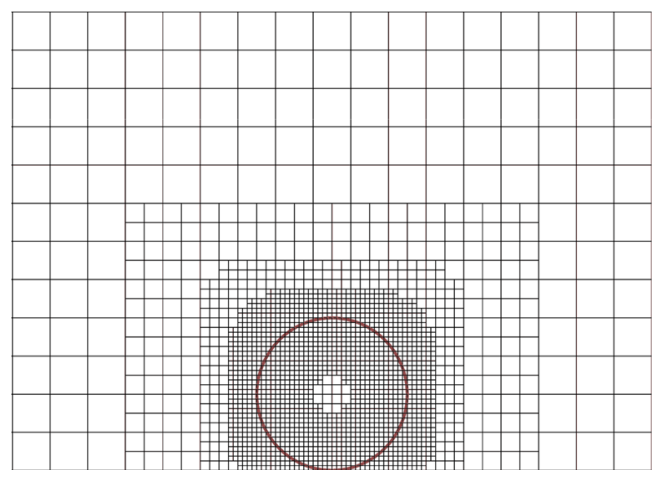

Fig. 2 Adaptive mesh refinement system

\section{Adaptive Mesh}

In the investigations of EHD flows, the three-dimensional numerical simulations need larger grid resolution and cost more computing time. In order to solve these problems, the adaptive mesh refinement (AMR) techniques are developed for the fluid flow equations and electric field equations. Figure 2 shows the adaptive mesh refinement system. The octree method is used to refine and coarsen the grids, and the grid resolution around the two-phase interfaces is largest. Farther away from the interfaces, the grid resolution is smaller, which can greatly decrease the number of grids and reduce the computing time. In the present study, the grid resolution $R / \Delta=16$ around the bubble is adopted due to the fact that Wang et al. (2015) assigned that 15 cells to the radius $R$ of a bubble rising in electric fields can obtain accurate results.

\section{Results and Discussions}

This section presents the numerical results of the effects of electric field intensity, permittivity, bubble initial position on bubble departing from the outside wall of a horizontal square tube in microgravity. To describe bubble behaviors, the following dimensionless parameters are adopted.

$$
\begin{aligned}
& R e=\frac{\rho_{l} \sqrt{R^{3} g}}{\mu_{l}}, B o=\frac{\rho_{l} g R^{2}}{\sigma}, B o_{e}=\frac{\varepsilon \varepsilon_{0} E_{0}^{2} R}{\sigma}, \lambda_{\rho}=\frac{\rho_{l}}{\rho_{g}}, \\
& \lambda_{\mu}=\frac{\mu_{l}}{\mu_{g}}, \lambda_{\varepsilon}=\frac{\varepsilon_{l}}{\varepsilon_{g}}
\end{aligned}
$$

where the Reynolds $(R e)$ number is the ratio of inertial force to viscous force, the gravitational Bond $(\mathrm{Bo})$ number the relative magnitude of gravity and interface tension force, and the electric Bond $\left(\mathrm{Bo}_{e}\right)$ number the ratio of electric force to interface tension force. The electric field $E_{0}$ is calculated by the electric potential $\psi_{0}$ of the outside wall of the small square tube, $E_{0}=\psi_{0} /\left(L_{z}-0.5 l\right)$. The $\lambda_{\rho}, \lambda_{\mu}$, and $\lambda_{\varepsilon}$ are the ratios of density, viscosity, and relative permittivity, respectively. In the following simulation, $\lambda_{\rho}$ and $\lambda_{\mu}$ are set to be 100. In sections "Results and Discussions", "Bubble Detachment and Rising in Different Gravity Condi tions $\left(B o_{e}=0\right)$ ", and "Bubble Detachment and Rising in Microgravity and Nonuniform Electric Fields (1)-(2)", the initial centroid of the bubble is $(0.05 \mathrm{~m}, 0.05 \mathrm{~m}, 0.014 \mathrm{~m})$.

\section{Bubble Detachment and Rising in Different Gravity Conditions $\left(\mathrm{Bo}_{e}=\mathbf{0}\right)$}

The effect of gravity on bubble dynamics in the absence of electric field is shown in Fig. 3. Figure 3a shows the bubble detachment and rising under terrestrial gravity condition ( $g=g_{0}=9.81 \mathrm{~m} / \mathrm{s}^{2}$ ), and the flow conditions are $R e=40$, $B o=2$. Initially, the spherical bubble attaches to the outside wall of the small square tube, and then the bubble rapidly departs from the tube outside wall due to the influence of larger buoyancy. Subsequently, the bubble rises in the liquid and gradually takes an oblate spheroid. Keeping the twophase fluid physical properties unchanged, bubble behaviors under microgravity conditions $\left(g=0.1 g_{0}, 0.01 g_{0}\right)$ are
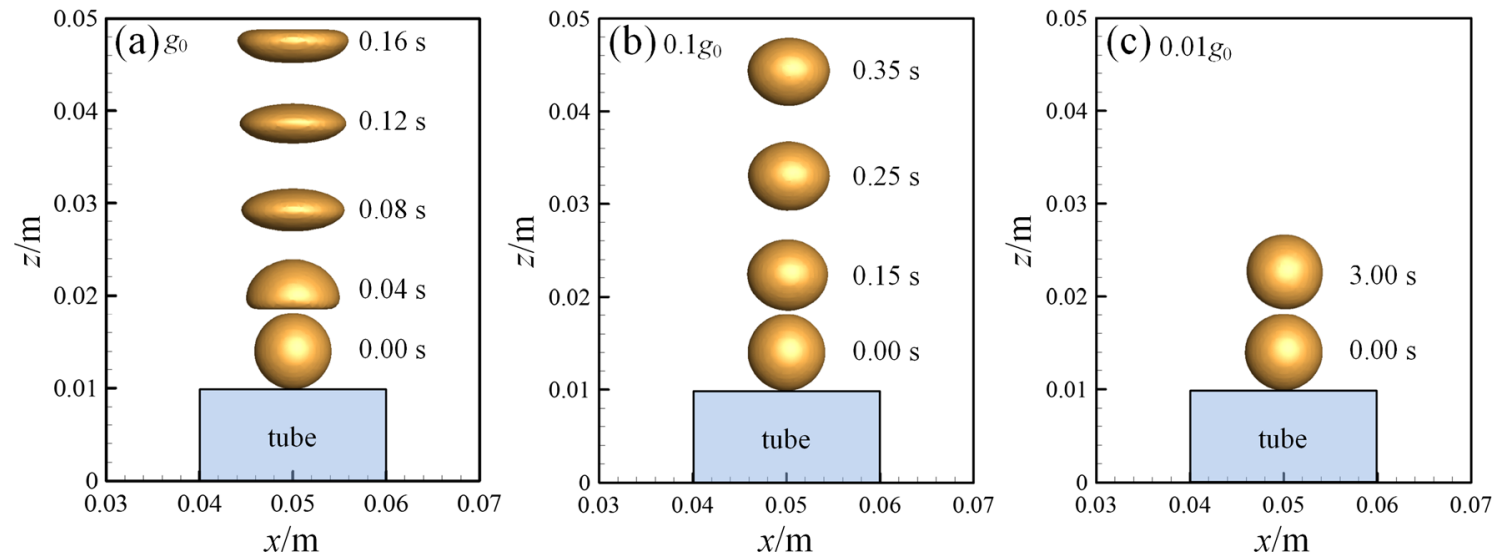

Fig. 3 Bubble detachment and rising in different gravity conditions 


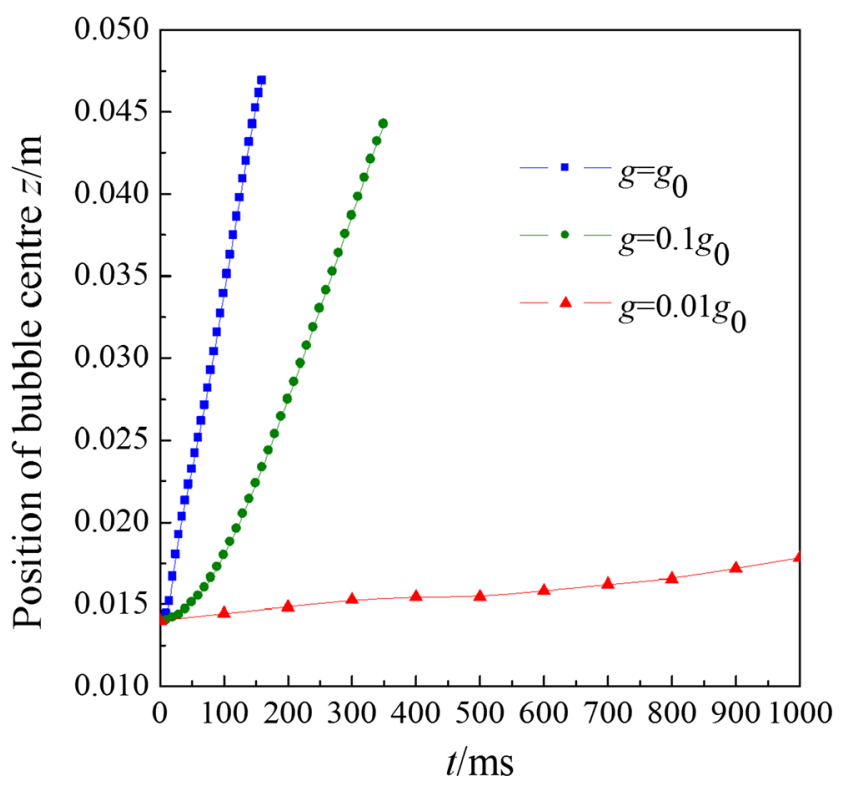

Fig. 4 Position of bubble centre versus time for different gravity conditions

shown in Fig. 3b and c, respectively. From Fig. 3a-c, it can be found that the decrease in gravity leads to the significant decrease in the effect of the buoyancy, and thus the bubble has smaller detachment and rising velocities. The bubble deformations in microgravity conditions are unobvious and approximately keep spherical shape. Figure 4 shows the position of the bubble centre changing with time for different gravity conditions. It can be seen in Fig. 4 that at $g=0.01 g_{0}$, the bubble stays near the tube wall for a very long time, which would make nucleate boiling develop into film boiling against heat transfer. In order to enhance heat transfer of nucleate boiling in microgravity conditions, an effective method for accelerating bubble detachment and rising is needed. In the following investigations, we try imposing nonuniform electric fields to control bubble deformation and motion in microgravity.

\section{Bubble Detachment and Rising in Microgravity and Nonuniform Electric Fields}

\section{(1) Influence of electric intensity}

In the present study, the nonuniform electric fields are established by two horizontal electrodes (as shown in Fig. 1), and the distributions of electric streamlines and electric field intensity $\left(|E|=\sqrt{E_{x}^{2}+E_{y}^{2}+E_{z}^{2}}\right)$ in the $x z$ plane are shown in Fig. 5. It can be seen that the electric field intensity in regions near the outside wall of the small square tube is higher than that in other regions, and the farther away from the small square tube, the smaller electric field intensity. It is anticipated that the nonuniformity of the electric field distribution would greatly affect the bubble deformation and motion. Figure 6a presents that the bubble detachment and rising processes in microgravity condition ( $\left.g=0.01 g_{0}=0.0981 \mathrm{~m} / \mathrm{s}^{2}\right)$ under the influence of a nonuniform electric field $\left(E_{0}=859.5 \mathrm{kV} / \mathrm{m}\right)$, and the flow conditions are $R e=4, B o=0.02, B o_{e}=1, \lambda_{\varepsilon}=3$. It can be found that when a nonuniform electric field is imposed on the flow field, the bubble is stretched along the direction of $z$ axis at the initial stage. When bubble departing from the tube wall, the bottom of the bubble rapidly shrinks due to the effect of surface tension force and electric force, and then the bubble gradually rises. Figure $6 \mathrm{~b}$ shows the the position of the bubble centre changing with time. It can be seen that the rising velocity of the bubble in the nonuniform electric field is larger than that in the absence of electric field. This case can be explained by the distributions of electric force on the bubble. Figure 7a shows the schematic diagram of the distribution of the electric force acted on the bubble, and the electric force calculated by Eq. 12 has the same direction with surface tension force due to the fact that the ratio of liquid permittivity to gas permittivity is greater than 1 . Figure $7 \mathrm{~b}$ presents that the electric field intensity around the bubble for different time in Fig. 6. The electric field intensity near the bottom of the bubble is higher than that near the top of the bubble, and therefore there is a larger electric
Fig. 5 Distributions of electric streamlines and electric field intensity in the $x z$ plane

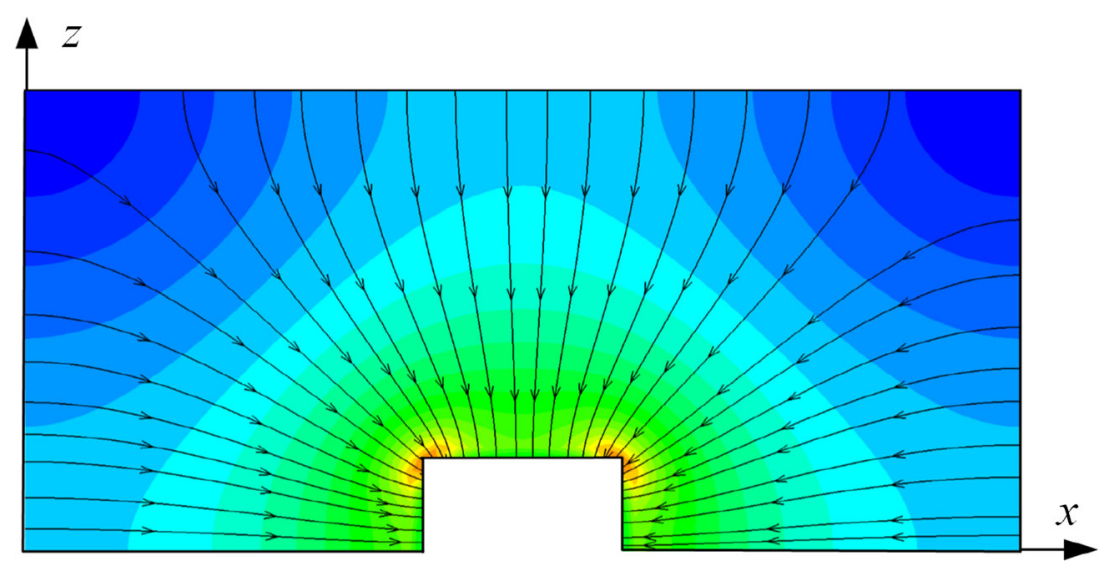


Fig. 6 Effect of nonuniform electric field on the bubble detachment and rising. a evolution of the bubble shape under the conditions of $R e=4$, $B o=0.02, B o_{e}=1, \lambda_{\varepsilon}=3$, $g=0.01 g_{0} ; \mathbf{b}$ position of bubble centre versus time for $B o_{e}=0,1$ with $g=0.01 g_{0}$
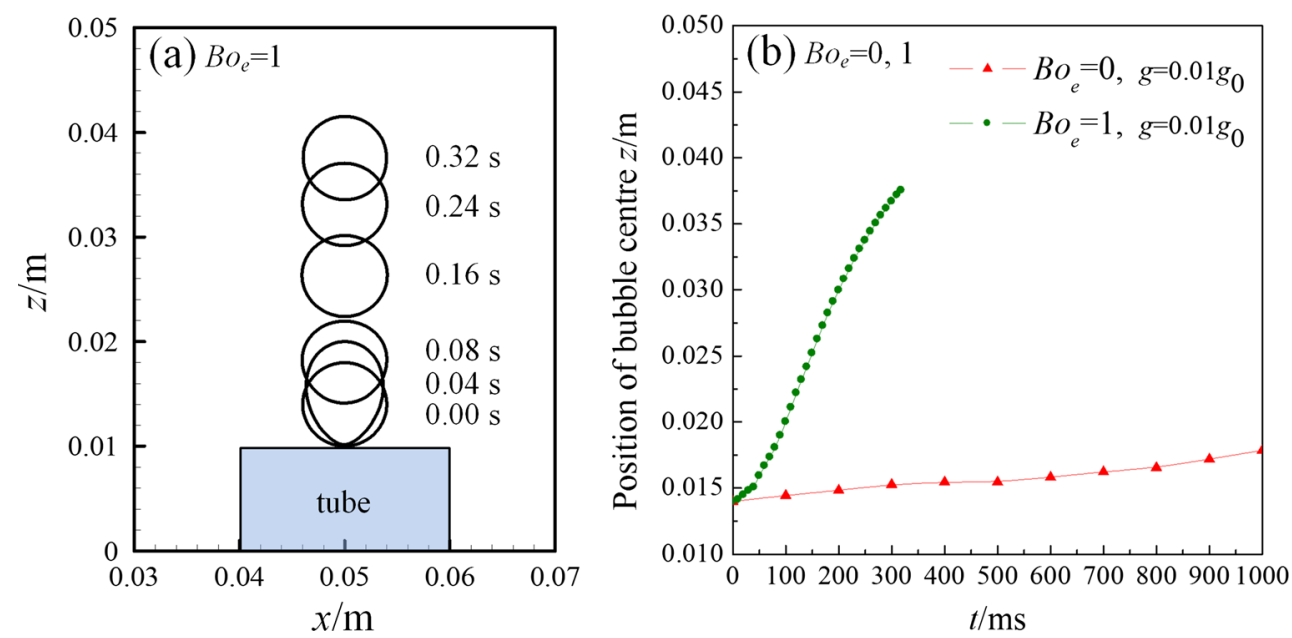

force on the bottom of the bubble. The vertical component of the total electric force acted on the bubble is positive, which drives bubbles away from the region of the stronger electric field.

Figure 8 shows the evolution of the bubble shape for other electric Bond numbers (corresponding electric field $\left.E_{0}=1488.6,1921.8,2717.8 \mathrm{kV} / \mathrm{m}\right)$ under the conditions of $R e=4, B o=0.02, \lambda_{\varepsilon}=3, g=0.01 g_{0}$. With the increase in the electric field intensity, the bubble deformation at the initial stage is more obvious. When the bubble departing from the wall, the larger electric field leads to the fact that the bottom of the bubble has larger shrink velocity caused stronger liquid jet in the wake of the bubble. And, the stronger liquid jet leads to the oscillatory deformation and break-up of the bubble under the effect of electric force, surface tension force, inertial force, and viscous force, see Fig. 8b-c. Figure 9a shows the position of bubble centre changing with time for different electric Bond number, and it can be seen that the increase in electric field intensity results in larger

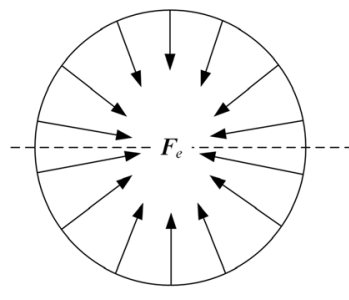

(a)

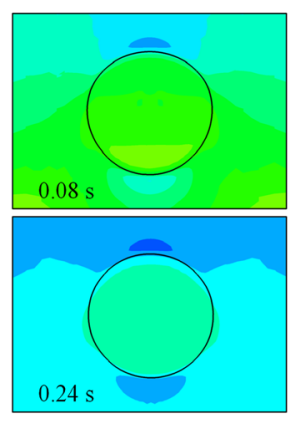

(b)

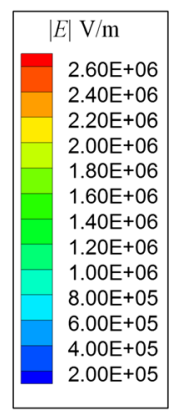
$2.40 \mathrm{E}+06$ $2.20 \mathrm{E}+06$ $.00 \mathrm{E}+06$
$80 \mathrm{E}+06$ $1.60 \mathrm{E}+06$ $1.40 \mathrm{E}+06$ $20 \mathrm{E}+06$ $1.00 \mathrm{E}+06$
$8.00 \mathrm{E}+05$ $6.00 \mathrm{E}+05$

$2.00 \mathrm{E}+05$

Fig. 7 Distributions of electric force and electric field intensity. a schematic diagram of the distribution of the electric force acted on the bubble; $\mathbf{b}$ distributions of electric field intensity around the bubble for different time in Fig. 6 bubble detachment and rising velocity due to the increase in the vertical component of the total electric force acted on the bubble. The vertical component of the total electric force can be calculated by the following formula.

$F_{\text {total }_{-}}=\sum_{i=0}^{N}\left(F_{S_{-} z}\right)_{i}$

where $F_{\text {total } z}$ is the vertical component of the total electric force, $N$ the number of cells contain phase interface, $F_{S_{-} z}$ the vertical component of electric force in a cell. The $F_{\text {total }} z$ versus the position of bubble centre for different electric Bond number are plotted in Fig. 9b. Initially, the $F_{\text {total_z }}$ decreases first and then increases with the increase of the position of bubble centre due to the deformation of the bubble. After bubble detachment, the bubble rising results in the increase in the distance between the bubble and the small square tube, and this case leads to the decrease in $F_{\text {total } z}$. At the same position, the larger electric field results in a larger driving force acted on the bubble.

\section{(2) Influence of permittivity}

Besides the electric field intensity, the ratio $\left(\lambda_{\varepsilon}\right)$ of liquid permittivity to gas permittivity is another important factor affecting the distribution and magnitude of electric force. The effect of $\lambda_{\varepsilon}$ on bubble detachment and rising is investigated by changing liquid permittivity. Figure 10 shows the evolution of the bubble shape for different $\lambda_{\varepsilon}$ with the conditions of $R e=4, B o=0.02, E_{0}=859.5 \mathrm{kV} / \mathrm{m}, g=0.01 g_{0}$. At the same electric field intensity, the larger $\lambda_{\varepsilon}$ leads to a larger bubble deformation, and the oscillatory deformation and break-up of the rising bubble also are found in Fig. 10b-c. Figure 11a shows the position of bubble centre changing with time for different $\lambda_{\varepsilon}$, and it can also be seen that the bubble detachment and rising velocity increases 

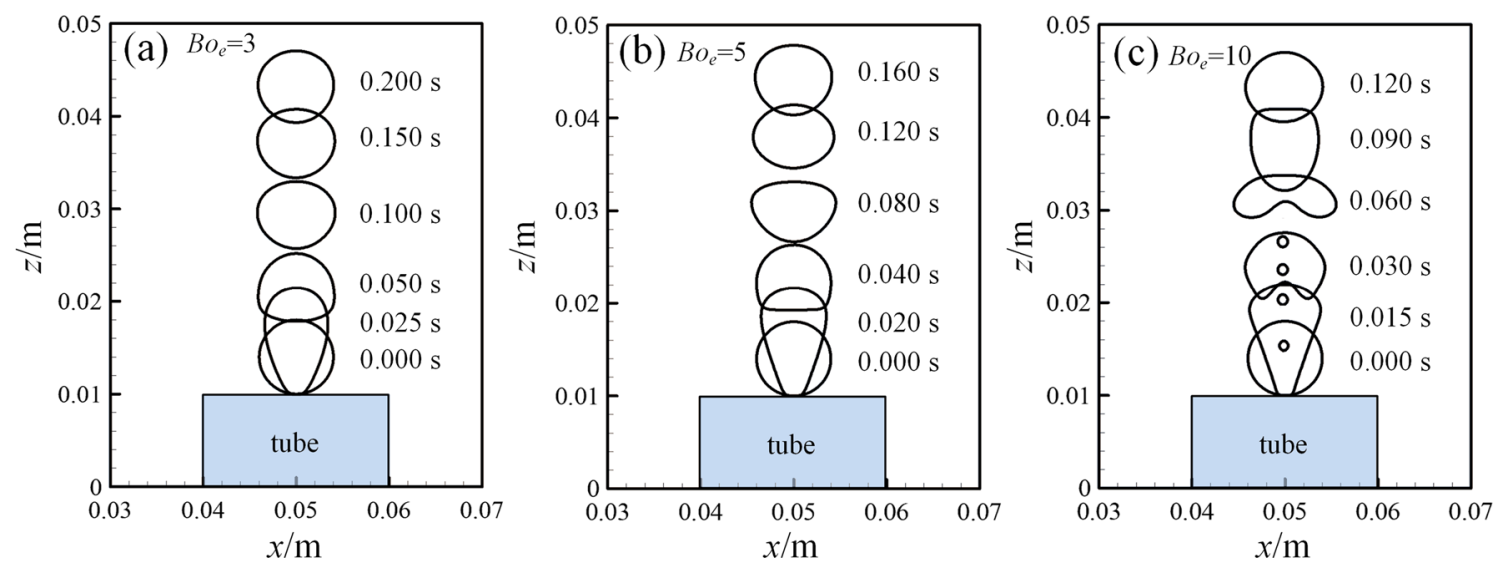

Fig. 8 Evolution of the bubble shape for different electric Bond number under the conditions of $\operatorname{Re}=4, B o=0.02, \lambda_{\varepsilon}=3, g=0.01 g_{0}$

Fig. 9 Bubble position and total electric force changing for different electric Bond number with $g=0.01 g_{0}$. a position of bubble centre versus time; $\mathbf{b}$ vertical component $\left(F_{\text {total }_{z}}\right)$ of the total electric force versus the position of bubble centre
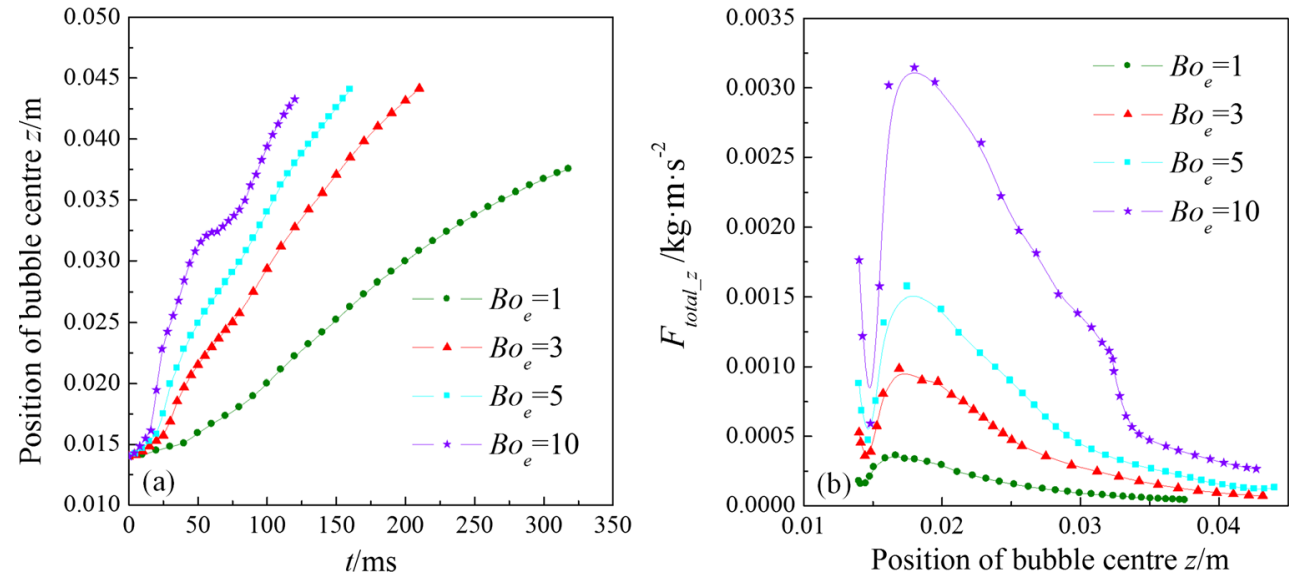
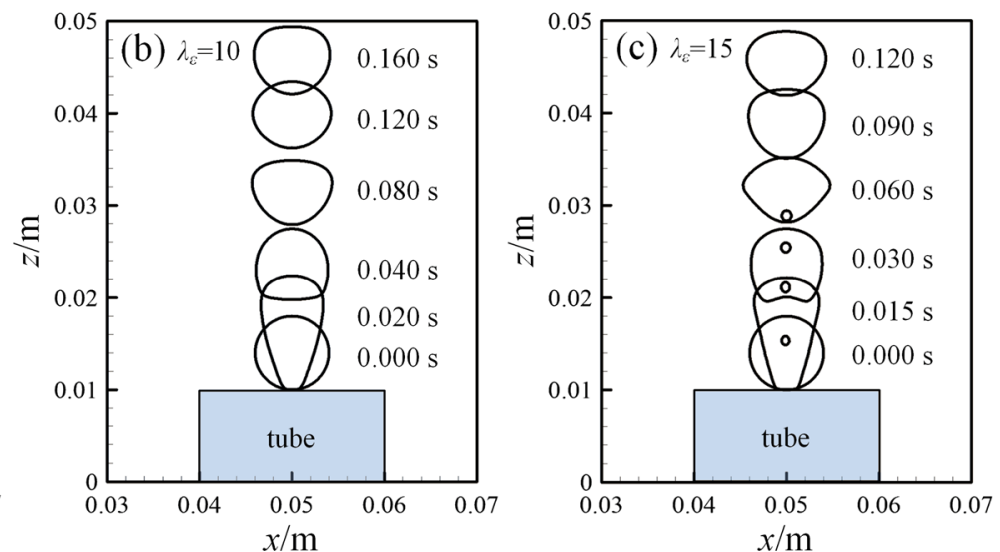

Fig. 10 Evolution of the bubble shape for different $\lambda_{\varepsilon}$ with the conditions of $R e=4, B o=0.02, E_{0}=859.5 \mathrm{kV} / \mathrm{m}, g=0.01 g_{0}$ 
Fig. 11 Bubble position and total electric force changing for different ratios of liquid permittivity to gas permittivity under the conditions of $R e=4$, $B o=0.02, E_{0}=859.5 \mathrm{kV} / \mathrm{m}$, $g=0.01 g_{0}$. a position of bubble centre versus time; $\mathbf{b}$ vertical component $\left(F_{\text {total_z }}\right)$ of the total electric force versus the position of bubble centre

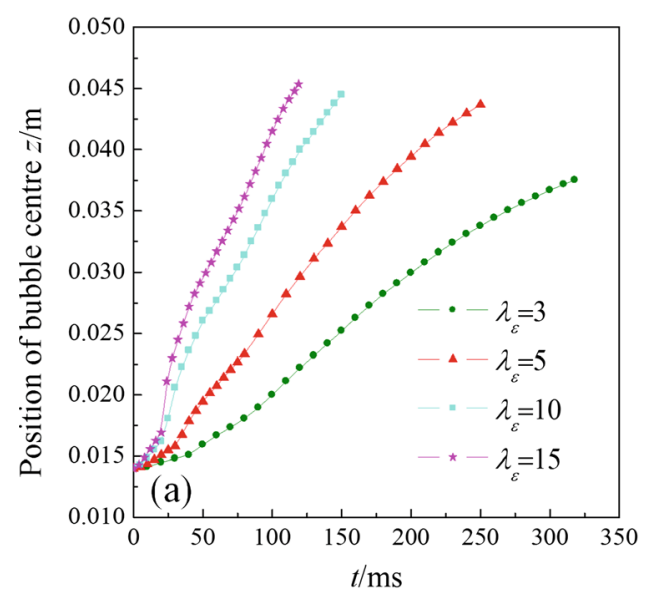

with the increase in $\lambda_{\varepsilon}$ which results in larger driving force acted on the bubble. The evolution of the vertical component $\left(F_{\text {total }} z\right)$ of the total electric force in Fig. $11 \mathrm{~b}$ are similar with that in Fig. 9, which is the reason that the effects of $\lambda_{\varepsilon}$ on bubble dynamics are similar with electric field intensity.

\section{(3) Influence of bubble initial position}

In the above investigations, electric fields around the bubble are symmetric about the planes of $x=0.05 \mathrm{~m}$ and $y=0.05 \mathrm{~m}$, which results in a symmetric bubble deformation. The rising trajectory of the bubble is a straight line due to the fact that the total electric force has no horizontal component. In this section, we study the influence of the asymmetric distribution of the nonuniform electric field on bubble deformation and motion by changing the bubble initial position. Figure 12 shows the evolution of the bubble shape and distributions of the electric field intensity around the bubble under the conditions of $R e=4, B o=0.02, B o_{e}=3$, $\lambda_{\varepsilon}=3, g=0.01 g_{0}$ with the bubble initial position ( 0.044 $\mathrm{m}, 0.05 \mathrm{~m}, 0.014 \mathrm{~m})$. Initially, the bottom left part of the bubble has an asymmetric deformation due to the fact that the electric field in the edge of the small square tube is the strongest, see Figs. 4 and 12b. The larger electric force at the bottom of the bubble drives the bubble departing from the tube wall. After bubble detachment, the rising trajectory of the bubble is a curve. From Fig. 10c, it can be seen that electric field intensity at the bottom and right of the bubble is stronger than that at the top and left of the bubble, which leads to the horizontal and vertical motions of the bubble. From the big square tube closer, the bubble has more obvious horizontal motion due to the larger horizontal gradient of electric field, see Fig. 4.

Figure 13 shows the evolution of bubble shape for other electric Bond number. Compared with Figs. 12 and 13, it can be seen that larger electric field intensity leads to more obvious deformation and break-up of the bubble. And, the increase in the electric field intensity can also result in larger
Fig. 12 Evolution of the bubble shape and distributions of electric field intensity around the bubble under the conditions of $R e=4, B o=0.02, B o_{e}=3$, $\lambda_{\varepsilon}=3, g=0.01 g_{0}$ with the bubble initial position $(0.044 \mathrm{~m}$, $0.05 \mathrm{~m}, 0.014 \mathrm{~m}$ )

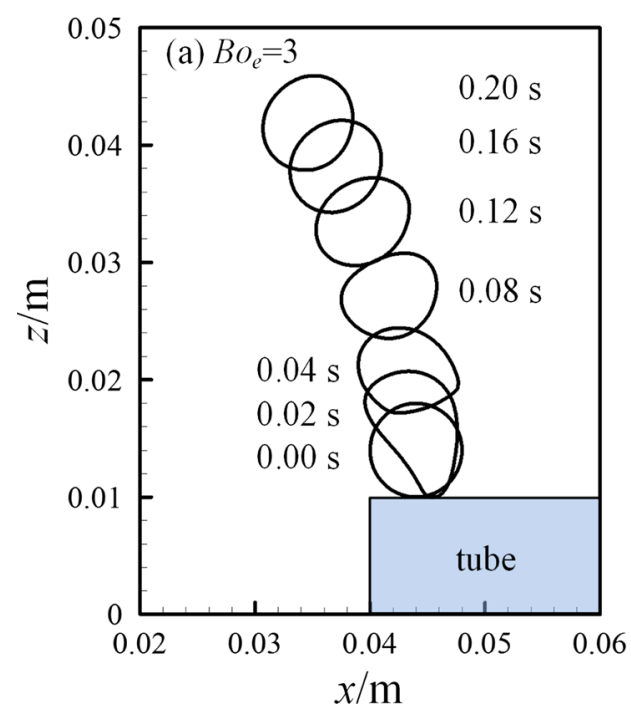

$|E| \mathrm{V} / \mathrm{m}$

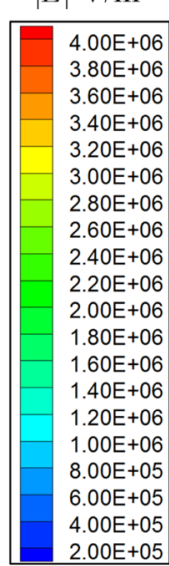


Fig. 13 Evolution of the bubble shape for different electric Bond number under the conditions of $R e=4, B o=0.02, \lambda_{\varepsilon}=3$, $g=0.01 g_{0}$ with the bubble initial position $(0.044 \mathrm{~m}, 0.05$ $\mathrm{m}, 0.014 \mathrm{~m})$
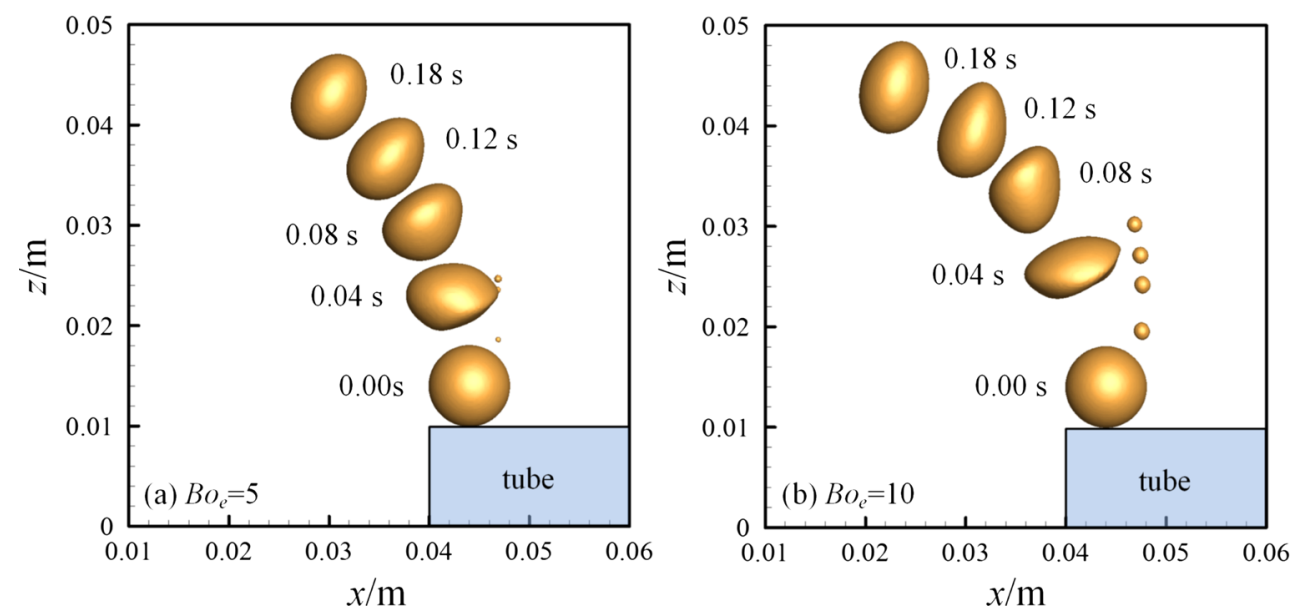

rising velocity and horizontal moving velocity of the bubble due to the larger horizontal and vertical driving force. In Fig. 13b, it can be found that the moving velocity of the small bubble is smaller than that of the big bubble, which indicates that the driving force acting on the big bubble is larger.

\section{Conclusions}

This paper presents a three-dimensional VOSET method for the numerical simulation of bubble dynamics in microgravity under the influence of nonuniform electric fields, in combination with the specially developed adaptive mesh refinement (AMR) techniques. The VOF advection equation, electric field equations, and fluid flow equations are solved on the adaptive octree grid system which can enhance the calculation accuracy and save the computer resources. Then, the effect of gravity, electric field intensity, fluid permittivity, and bubble initial position on bubble detachment and rising are investigated. The conclusions from the numerical results can be summarized as follows:

1. In terrestrial gravity condition, the bubble can rapidly depart from the tube wall and rise in the liquid due to the influence of buoyancy. However, the buoyancy for microgravity conditions is weak or lacking, which goes against bubble detachment and rising.

2. Nonuniform electric fields can supply an additional driving force as a replacement of the buoyancy to accelerate the bubble detachment and rising. The electric force would drive bubbles away from the region of stronger electric field. When increasing electric field intensity or the ratio of fluid permittivity to gas permittivity, the bubble has a larger deformation and detachment/rising velocities.
3. When the nonuniform electric field around the bubble is asymmetric, the rising velocity of the bubble has a horizontal component, thus the rising trajectory of the bubble is not a straight line. And, the asymmetric distribution of the electric force leads to the irregular bubble deformation.

Acknowledgments The present study is supported financially by the National Natural Science Foundation of China (No.51176153 and 11372327) and the Strategic Priority Research Program on Space Science, the Chinese Academy of Sciences under the grant of XDA04020404.

\section{References}

Annaland, M.V., Deen, N.G., Kuipers, J.A.M.: Numerical simulation of gas bubbles behaviour using a three-dimensional volume of fluid method. Chem. Eng. Sci. 60, 2999-3011 (2005)

Bari, S.D., Robinson, A.J.: Adiabatic bubble growth in uniform DC electric fields. Exp. Therm. Fluid Sci. 44, 114-123 (2013)

Brackbill, J.U., Kothe, D.B., Zemach, C.: A continuum method for modeling surface tension. J. Comput. Phys. 100, 335-354 (1992)

Castellanos, A.: Electrohydrodynamics. Springer-Verlag Wien, New York (1998)

Di Marco, P.: Review of reduced gravity boiling heat transfer: European research. J. Jpn. Soc. Microgravity Appl. 20, 252-263 (2003a)

Di Marco, P., Grassi, W., Memoli, G., Takamasa, T., Tomiyama, A., Hosokawa, S.: Influence of electric field on single gas-bubble growth and detachment in microgravity. Int. J. Multiphase Flow 29, 559-578 (2003b)

Di Marco, P., Grassi, W.: Pool boiling in microgravity and in electric fields: old and recent results. Multiphase Sci. Technol. 19, 141165 (2007)

Di Marco, P.: The use of electric force as a replacement of buoyancy in two-phase flow. Microgravity Sci. Technol. 24, 215-228 (2012a)

Di Marco, P.: Influence of force fields and flow patterns on boiling heat transfer performance: a review. J. Heat Transfer 134, 030801 (2012b) 
Dong, W., Li, R.Y., Yu, H.L., Yan, Y.Y.: An investigation of behaviours of a single bubble in a uniform electric field. Exp. Therm. Fluid Sci. 30, 579-586 (2006)

Gao, M., Cheng, P., Quan, X.: An experimental investigation on effects of an electric field on bubble growth on a small heater in pool boiling. Int. J. Heat Mass Transfer 67, 984-991 (2013)

Herman, C., Iacona, E., Foldes, I.B., Suner, G., Milburn, C.: Experimental visualization of bubble formation from an orifice in microgravity in the presence of electric fields. Exp. Fluids 32, 396412 (2002)

Herman, C., Iacona, E.: Modeling of bubble detachment in reduced gravity under the influence of electric fields and experimental verification. Heat Mass Transfer 40, 943-957 (2004)

Hu, W.R., Zhao, J.F., Long, M., Zhang, X.W., Liu, Q.S., Hou, M.Y., Kang, Q., Wang, Y.R., Xu, S.H., Kong, W.J., Zhang, H., Wang, S.F., Sun, Y.Q., Hang, H.Y., Huang, Y.P., Cai, W.M., Zhao, Y., Dai, J.W., Zheng, H.Q., Duan, E.K., Wang, J.F.: Space program SJ-10 of microgravity research. Microgravity Sci. Technol. 26, 159-169 (2014)

Iacona, E., Herman, C., Chang, S., Liu, Z.: Electric field effect on bubble detachment in reduced gravity environment. Exp. Therm. Fluid Sci. 31, 121-126 (2006)

Jalaal, M., Khorshidi, B., Esmaeilzadeh, E., Mohammadi, F.: Behavior of a single bubble in a nonuniform DC electric field. Chem. Eng. Commun. 198, 19-32 (2010)

Jaworek, A., Sobczyk, A.T.: Electrospraying route to nanotechnology: An overview. J. Electrostat. 66, 197-219 (2008)

Kim, J.: Review of reduced gravity boiling heat transfer: US research. J. Jpn. Soc. Microgravity Appl. 20, 264-271 (2003)

Kim, J.: Review of nucleate pool boiling bubble heat transfer mechanisms. Int. J. Multiphase Flow 35, 1067-1076 (2009)

Lee, J., Moon, H., Fowler, J., Schoellhammer, T., Kim, C.-J.: Electrowetting and electrowetting-on-dielectric for microscale liquid handling. Sensor. Actuat, A-Phys. 95, 259-268 (2002)

Mahlmann, S., Papageorgiou, D.T.: Buoyancy-driven motion of a two-dimensional bubble or drop through a viscous liquid in the presence of a vertical electric field. Theor. Comput. Fluid Dyn. 23, 375-399 (2009)
Minemawari, H., Yamada, T., Matsui, H., Tsutsumi, J.Y., Haas, S., Chiba, R., Kumai, R., Hasegawa, T.: Inkjet printing of singlecrystal films. Nature 475, 364-367 (2011)

Ohta, H.: Review of reduced gravity boiling heat transfer: Japanese research. J. Jpn. Soc. Microgravity Appl. 20, 272-285 (2003)

Paknemat, H., Pishevar, A.R., Pournaderi, P.: Numerical simulation of drop deformations and breakup modes caused by direct current electric fields. Phys. Fluids 24, 26 (2012)

Straub, J.: Boiling heat transfer and bubble dynamics in microgravity. Adv. Heat Transfer 35, 57-172 (2001)

Sun, D.L., Tao, W.Q.: A coupled volume-of-fluid and level set (VOSET) method for computing incompressible two-phase flows. Int. J. Heat Mass Transfer 53, 645-655 (2010)

Sunder, S., Tomar, G.: Numerical simulations of bubble formation from submerged needles under non-uniform direct current electric field. Phys. Fluids 25, 102104 (2013)

Theodorakakos, A., Bergeles, G.: Simulation of sharp gas-liquid interface using VOF method and adaptive grid local refinement around the interface. Int. J. Numer. Methods Fluids 45, 421-439 (2004)

Wang, T., Li, H., Zhang, Y., Shi, D.: Numerical simulation of bubble dynamics in a uniform electric field by the adaptive 3DVOSET method. Numer. Heat. Transfer A-Appl. 67, 1352-1369 (2015)

Wang, Z., Yang, J., Koo, B., Stern, F.: A coupled level set and volume-of-fluid method for sharp interface simulation of plunging breaking waves. Int. J. Multiphase Flow 35, 227-246 (2009)

Xue, Y.-F., Zhao, J.-F., Wei, J.-J., Li, J., Guo, D., Wan, S.-X.: Experimental Study of Nucleate Pool Boiling of FC-72 on Smooth Surface under Microgravity. Microgravity Sci. Technol. 23, S75-S85 (2011)

Youngs, D.L.: Time-dependent multi-material flow with large fluid distorion. In: Morton, K.W., Baines, M.J. (eds.) Numerical Methods for Fluid Dynamics. Acadimic Press, New York (1982)

Yu, Z., Fan, L.S.: An interaction potential based lattice Boltzmann method with adaptive mesh refinement (AMR) for two-phase flow simulation. J. Comput. Phys. 228, 6456-6478 (2009)

Zhao, J.F.: Two-phase flow and pool boiling heat transfer in microgravity. Int. J. Multiphase Flow 36, 135-143 (2010) 